\title{
Distribution Voltage Control Considering the Impact of PV Generation on Tap Changers and Autonomous Regulators
}

Yashodhan P Agalgaonkar, Student Member IEEE, Bikash C Pal, Fellow IEEE and Rabih A Jabr, Senior Member IEEE

\begin{abstract}
The uptake of variable Mega Watts(MW) from Photovoltaics(PV) challenges distribution system operation. The primary problem is significant voltage rise in the feeder that forces existing voltage control devices such as on-load tapchangers and line voltage regulators to operate continuously. The consequence is the deterioration of the operating life of the voltage control mechanism. Also, conventional non-coordinated reactive power control can result in the operation of the line regulator at its control limit (runaway condition). This paper proposes an optimal reactive power coordination strategy based on the load and irradiance forecast. The objective is to minimize the number of tap operations so as not to reduce the operating life of the tap control mechanism and avoid runaway. The proposed objective is achieved by coordinating various reactive power control options in the distribution network while satisfying constraints such as maximum power point tracking of $P V$ and voltage limits of the feeder. The option of voltage support from PV plant is also considered. The problem is formulated as constrained optimization and solved through the interior point technique. The effectiveness of the approach is demonstrated in a realistic distribution network model.
\end{abstract}

Index Terms-PV, distribution networks, voltage control, reactive power optimization.

\section{INTRODUCTION}

The proportion of PV generation into existing power system generation mix has significantly increased in recent times. The PV has cumulative global installation of $70 \mathrm{GW}$ with $21.9 \mathrm{GW}$ of new installation in Europe alone in 2011 [1]. Most of the PV plants are getting connected to low/medium voltage distribution level as Distributed Generation(DG). However, this has led to many problems from the power system operation perspective. One of the operational problems is the variable voltage rise on distribution feeders due to DG [2]. The overvoltage can be managed by curtailing active power injection [3]. In the low voltage network this approach is required due to lower $\mathrm{X} / \mathrm{R}$ ratio [4] [5]. Though necessary, this directly results in suboptimal capture of energy from DGs.

Manuscript received November 17, 2012; revised April 03, 2013; accepted August 21, 2013

This work has been supported by a joint UK-India initiative in solar energy through a project 'Stability and Performance of Photovoltaic (STAPP)' funded by Research Councils UK (RCUK) Energy Programme in UK (contract no: EP/H040331/1) and by Department of Science and Technology (DST) in India. Y. Agalgaonkar and B. C. Pal are with the Department of Electrical and Electronic Engineering, Imperial College, London SW7 2AZ, U.K. e-mail: (yashodhan.a@imperial.ac.uk; b.pal@imperial.ac.uk). R. A. Jabr is with the Department of Electrical and Computer Engineering, American University of Beirut, Beirut 1107 2020, Lebanon (email:rj30@aub.edu.lb)
For instance, the German grid has $22 \mathrm{GW}$ of solar. However, because of the voltage rise issue only $70 \%$ of the total capacity is paid through feed-in-tariff for plants smaller than $30 \mathrm{kWp}$. The result is that PV owners are discouraged to operate their plants at full capacity [6]. With voltage source converters interfaced with DG, the option of reactive power control is available in medium voltage(MV) network [7]. Though till now PV operation is at unity power factor, some of the technical standards [8] [9] are supportive of this feature to be exploited giving PV plants the opportunity to participate in voltage/reactive power control.

The Distribution Network Operators(DNOs) maintain appropriate voltage profile across the network with the help of on load tap changers(OLTCs), voltage regulators(VRs) and capacitors. In most of the distribution feeders these devices are controlled based on the local signals, for example, bus voltage for OLTC and VR and time control or local bus voltage control for capacitors. In this conventional voltage control method all devices operate autonomously in a non coordinated manner. This non coordinated approach and unity power factor PV generation impacts the operation of OLTCs and VRs. The presence of PV on distribution feeders will lead to increased number of OLTC and VR operations and wear and tear of the devices [10], [11]. The result is an increase in the OLTC and VR maintenance/overhaul cost incurred by the DNOs. Under the scenario of significant power injection by PV, power flows can reverse on a distribution feeder. Some OLTC construction is such that permissible reverse power flow is less than the apparent power rating of the transformer [12]. Under specific operational scenario VR fails to control voltage at the regulated bus and reaches lowest or highest tap limit. This phenomenon is widely referred to as 'reverse power tap changer runaway' condition [13] [14]. This highlights the fact that in the presence of PV on the feeder, an appropriate consideration of OLTC, VR and their control settings are necessary for an effective voltage control strategy. Most of the recent approaches replace local autonomous control of the voltage control devices with communication based supervisory control [15] [16]. In [15] voltage control devices are remotely dispatched on an hourly basis through a communication channel to achieve flat voltage profile in the system without DG. Unity power factor PV in distribution systems is considered in [16]. Further, two ways communication based real time smart grid Volt-Var control is proposed in [17]. Though the above mentioned schemes will certainly be able to alleviate the challenges, they require 


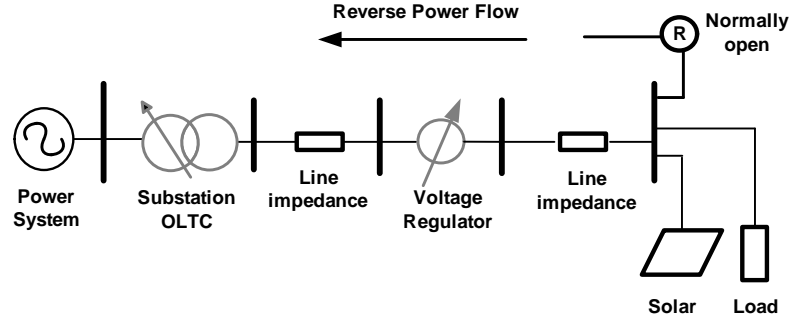

Figure 1. Radial system topology

communication with all voltage control devices. However the operation of VRs is still autonomous based on local signals, without communication links on many distribution feeders [18]. The switched capacitors also lack communication links on some feeders and timer control is still operational in Europe [19] [20]. Considering this fact in [19], a combined local and remote voltage control strategy is discussed. It proposed that by opting complete remote control of voltage control devices, they could loose their ability to react to load changes. A local communication-less Volt-Var control in the presence of synchronous machine based DG is considered in [20]. Most of the available literatures do not consider detailed impact of DG such as PV on autonomous local control setting of VR. It is also important to have an insight into what possible interaction a PV and its reactive power settings will have with the autonomous operation of a VR.

In this work, the detailed impact of PV on VR and OLTC is considered. A coordination strategy is proposed to alleviate the negative impact on OLTC and VR using reactive power option from PV plant. Following this introduction, Section II describes details of voltage control devices in the distribution system. Section III details coordination between PV and VR and discusses operational challenges. Section IV describes the optimal coordination strategy. Section V presents a case study and presents results. Section VI summarizes contributions and conclusions of the work.

\section{Voltage Control Devices}

Fig.1 shows a MV radial distribution system diagram. This has PV along with its reactive power control capability. The other voltage control devices are OLTC and VR. The VR operates in an autonomous mode. It consists of normally open switch which connects to an alternate power feeding point.

\section{A. Voltage support from $P V$}

A grid integrated PV model is shown in Fig.2. The converter control achieves the best possible active power capture through maximum power point tracking(MPPT). As shown in Fig. 2, $\mathrm{PV}$ also offers voltage support by controlling reactive power at the point of common coupling (PCC). The reactive power output and hence PCC voltage control capability of PV will be limited by the inverter apparent power rating. The capability curve in Fig. 3 shows the real and reactive power output possible from the PV plant. In order to give reactive power support at peak active power injection, overcapacity of the inverter is necessary as sketched in Fig. 3.

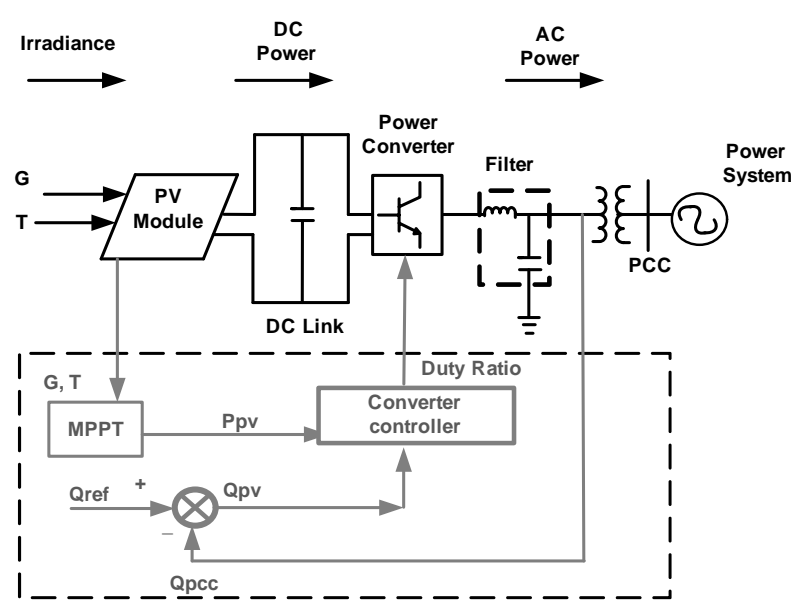

Figure 2. Generic grid integrated PV topology

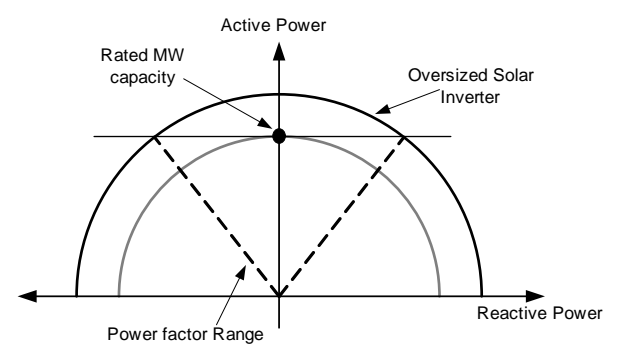

Figure 3. Solar generation capacity curve

The DNOs have an option of setting fixed reactive power or fixed power factor of PV plant as real power varies. The operator also has an option to define variable power factor as active power varies (power factor(P)), or variable reactive power as voltage varies $(\mathrm{Q}(\mathrm{U}))$. PV connected to a strong substation bus is controlled more effectively in these reactive power or power factor control modes. Depending on the capacity of PV plant and fault level at PCC, PV can be operated in voltage control mode. The PV can regulate the voltage of a weak bus having lower fault level. Thus voltage variation will be compensated by PV reactive power support.

Fig. 4 is simple equivalent circuit showing PCC voltage control by PV plant. In Fig. $4 V_{1}$ is constant reference voltage. Voltage drop $\Delta V$ is given by,

$$
\Delta V=\frac{R\left(P_{\text {load }}-P_{p v}\right)+X\left(Q_{\text {load }} \pm Q_{p v}\right)}{V_{2}}
$$

PV generation MPPT active power is denoted by $P_{p v}$ and reactive power output is $Q_{p v}$. Load power is denoted by $P_{l o a d}$ and $Q_{\text {load. }} R+j X$ represent impedance of the line. So any

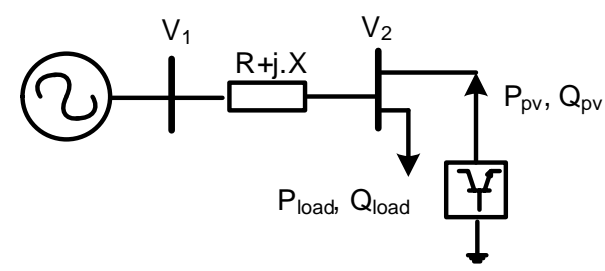

Figure 4. PCC voltage control by PV plant 


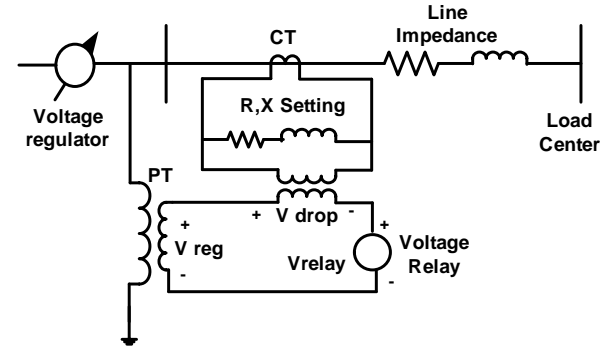

Figure 5. Line drop compensation [22]

variation in voltage $V_{2}$ and hence $\Delta V$ due to $P_{\text {load }}, Q_{\text {load }}$ and $P_{p v}$ can be mitigated by varying $Q_{p v}$ to either inductive or capacitive value. Thus PCC voltage $V_{2}$ can be controlled or kept constant by the PV plant [19] [21].

\section{B. Voltage Regulator}

VR is typically an autotransformer with $\pm 10 \%$ voltage regulation capability. Fig.5 shows, the voltage regulation mechanism to compensate the voltage drop in the feeder between the source and load for different load current levels. This is widely known as line drop compensator. The details of the operating principle, settings and compensating impedance are available in [22]. The VR maintains the load terminal voltage at set value by adjusting the tap position as the load current varies.

Fig. 6 shows the equivalent circuit of MV distribution feeder. In a situation where part of the load is supplied by PVplant downstream, the situation changes. Particularly at high PV penetration, power flows in the reverse direction towards the source substation. This causes rise in the load voltage as can be seen from vector diagram in Fig. 7. The voltage control challenge will be more complex where PV plant has a reactive power control option. There will be interaction between VR and PV voltage control. The nature of the control interaction between VR and PV and its overall effectiveness depends on various factors such as the driving point impedance, PV capacity, PV reactive power setting, VR setting, and system topology. Table I summarizes generic interactions between VR autonomous mode and PV reactive power setting [14] [23] [24]. It is not difficult to appreciate that for low driving point impedance i.e. strong voltage source there is real risk of VR hitting its limit or operating in runaway condition thus compromising its control effectiveness. The details of the operational challenges are discussed next with reference to the model feeder topology of Fig. 1.

\section{Operational CHALLENGES}

There are primarily two autonomous operational modes of VR where they can potentially interact with PV voltage control action.

Bidirectional mode: In this mode the VR will switch its target voltage control node depending on the direction of active power flow through it. In the context of Fig. 6 during forward active power flow VR regulates $V_{3}$ or $V_{4}$ and during reverse active power flow VR regulates $V_{2}$. This VR mode is useful

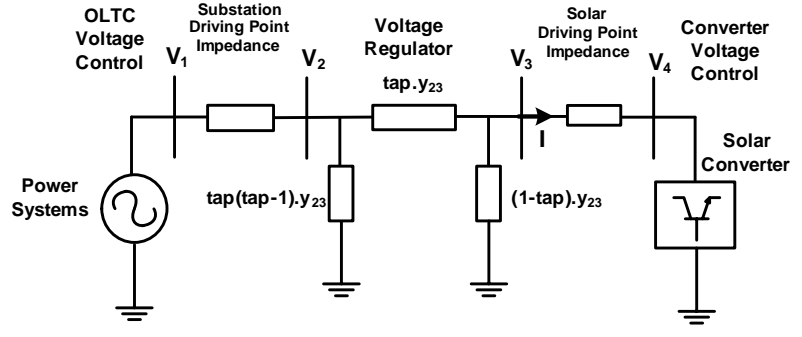

Figure 6. Equivalent circuit of a radial system with VR

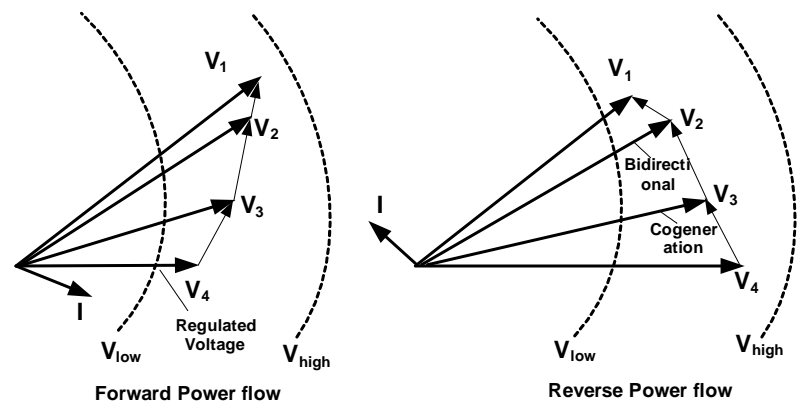

Figure 7. Vector diagram of a radial system

when alternate feed is activated by changing the status of 'normally open' (NO) switch to 'normally close' (NC) as shown in Fig.1. In the 'NO' position of switch, unity power factor PV can cause a reverse power flow through VR. This will result in the VR controlling voltage $V_{2}$ on the substation side. If the substation is the stronger source, i.e. with low driving point impedance, VR fails to set the required voltage set point and reaches the highest or lowest tap position; i.e. runaway condition. Under this situation the VR is ineffective in providing voltage regulation that will result in voltages $V_{3}$ and $V_{4}$ outside the prescribed limit on the side of PV. In a scenario when the substation side driving point impedance is higher and PV is capable of supplying reactive power, the runaway situation can be avoided. However it should be noted that PV having a reactive power capability is necessary but not sufficient. The PV should inject the optimum value of reactive power and target optimum voltage setpoint $V_{4}$ so that the VR does not operate close to its limit. Any positive voltage control contribution from the VR only helps to run the PV with reduced losses because of reduced reactive current.

Co-generation mode : In this mode the line drop compensator settings are altered at the time of reverse power flow. During forward power flow the VR regulates voltage $V_{4}$. During reverse power flow it regulates voltage $V_{3}$. When voltage control option from a large PV plant is available the VR should always regulate $V_{3}$ in this mode as it will have little influence on the voltage $V_{4}$. High capacity PV with lower driving point impedance can even drive the VR into a runaway situation while the VR is controlling the voltage $V_{3}$. In this mode, regardless of the direction of active power flow the VR continues to regulate the voltage in the forward direction that is $V_{3}$ or $V_{4}$. In the context of Fig.1, where alternate feed is activated by changing $\mathrm{NO}$ switch to $\mathrm{NC}$, this mode will still continue to regulate voltage $V_{3}$ which is 
Table I

INTERACTION BETWEEN PV REACTIVE POWER SUPPORT MODE AND VR AUTONOMOUS SETTING

\begin{tabular}{|l|l|l|l|}
\hline VR & Unity power factor & $\begin{array}{l}\text { Constant Q or power factor, } \\
\text { power factor(P) or Q(U) }\end{array}$ & \multicolumn{1}{|c|}{ Constant Voltage } \\
\hline Bidirectional & $\begin{array}{l}\text { Caution: Runaway during re- } \\
\text { verse power flow if low substa- } \\
\text { tion driving point impedance. } \\
\text { verse power flow if low sub- } \\
\text { station driving point impedance } \\
\text { and no optimum Q from PV. }\end{array}$ & $\begin{array}{l}\text { Caution: Runaway during re- } \\
\text { station driving point impedance } \\
\text { and no optimum voltage set } \\
\text { point from PV. }\end{array}$ \\
\hline $\begin{array}{l}\text { Reactive Bidirectional } \\
\text { manufacturers) }\end{array}$ & $\begin{array}{l}\text { Mostly for loop distribution } \\
\text { systems. No impact on reactive } \\
\text { current setting of VR due to PV. }\end{array}$ & $\begin{array}{l}\text { Mostly in loop distribution sys- } \\
\text { tems. Caution: Reactive cur- } \\
\text { rent set point of VR can be } \\
\text { impacted by PV. }\end{array}$ & $\begin{array}{l}\text { Mostly in loop distribution sys- } \\
\text { tems. Caution: Reactive cur- } \\
\text { rent set point of VR can be } \\
\text { impacted by PV. }\end{array}$ \\
\hline Co-generation & $\begin{array}{l}\text { Caution: Interference with the } \\
\text { line drop compensator setting. } \\
\text { line drop compensator settings }\end{array}$ & $\begin{array}{l}\text { Caution: Runaway for for- } \\
\text { ward/reverse active power flow } \\
\text { if PV driving point impedance } \\
\text { is low for high capacity PV. }\end{array}$ \\
\hline
\end{tabular}

not appropriate from the feeder operation point of view. The operational requirements with respect to OLTC and VR in the presence of PV are summarized as :

- The VR should not run away under any system scenario described in Table I.

- The voltage control in distribution system has to be achieved with minimum tap counts of OLTC and VR. This requires optimal control coordination.

\section{Optimal COORDinAtion}

\section{A. Control Strategy}

With reference to the equivalent circuit of Fig. 6 one possible situation can be that the PV is of high capacity and both sources, substation and $\mathrm{PV}$, are controlling voltages $V_{1}$ and $V_{4}$ respectively. Usually field settings should be such that the weaker source (higher driving point impedance values) side voltage is regulated by the VR. For weaker substation source, during reverse power flow, the VR will be set to regulate $V_{2}$. During forward power flow, for the cases with weaker PV source, VR can regulate $V_{3}$ effectively. $V_{1}$ and $V_{4}$ are tightly regulated by substation OLTC and large PV plant when both sources are stronger (smaller driving point impedance values), and in that situation the VR will neither be able to control $V_{2}$ nor $V_{3}$. However, the VR will still be useful for reactive power flow control. By varying the tap value, the shunt element $(1-\operatorname{tap}) y_{23}$ and $\operatorname{tap}(\operatorname{tap}-1) y_{23}$ will change their inductive or capacitive reactance. This will make the PV plant and substation OLTC to alter their reactive power injections. From Fig. 6, it can be seen that where both the PV and substation will have realistic driving point impedance values, VR runaway can be avoided by adjustment of OLTC bus voltage $V_{1}$ and PV bus voltage $V_{4}$. This is achieved through coordinated and optimum voltage set point adjustment of OLTC and PV.

\section{B. Optimization}

The coordination between PV reactive power control capability and voltage control option of OLTC and VR is an optimization problem and its goal is to contain excessive/unnecessary operations of OLTC and VR and avoid VR runaway situation. This is formulated in this section as optimal reactive power dispatch problem subject to meeting the various network operational constraints.

1) Objective function: The objective is to minimize the total number of tap operations over the 24 hours; subject to various equality and inequality constraints

$$
\text { Minimize } f_{\text {count }}=\sum_{t=1}^{t=N} \sum_{T=1}^{T=N_{t r}}\left|\operatorname{Tap}_{t, T}-\operatorname{Tap}_{t-1, T}\right|
$$

where $t$ is time instant, $N$ number of time instants considered in a day. The total number of time instants $N$ will depend on the size of the time interval between instants $t$ and $t+1$. T is the transformer number, $N_{t r}$ is total number of OLTCs and VRs. The constraint related to VR runaway is included as a penalty in the objective function which is discussed later.

2) Constraints: Different equality and inequality constraints are as follows:

Current balance: Equality constraints are represented by a set of nodal current injection equations formulated in rectangular coordinates. For generic load bus $l$ connected with generation bus $g$, the mathematical model and Newton Raphson algorithm can be represented in schematic form as,

$$
\left[\begin{array}{c}
\Delta I_{i m c}^{a b c} \\
\Delta I_{r e l}^{a b c} \\
\Delta I_{i m g}^{a b c} \\
\Delta I_{r e g}^{a b c}
\end{array}\right]=J\left[\begin{array}{c}
\Delta V_{r e l}^{a b c} \\
\Delta V_{i m c}^{a b c} \\
\Delta V_{i m g}^{a b c} \\
\Delta Q_{g}^{a b c}
\end{array}\right]
$$

In (3) $\Delta I_{r e}^{a b c}$ and $\Delta I_{i m}^{a b c}$ are real and imaginary current mismatch, $\Delta V_{r e}^{a b c}$ and $\Delta V_{i m}^{a b c}$ are real and imaginary voltage mismatch, and $J$ is the Jacobian. The detailed algorithm of the current injection method and modeling of voltage control devices are formulated as per reference [25] and [26].

Voltage limits: Steady state voltage at all the buses in the distribution system must be maintained within the prescribed limits.

$$
V_{\text {low }} \leq V_{t} \leq V_{\text {high }}
$$

Branch flow constraints: In steady state the maximum amount of current flow on the line is constrained by feeder thermal limit, hence for each feeder,

$$
I_{t} \leq I_{\max }
$$


Transformer capacity constraints: The maximum amount of apparent power flow through a transformer will be limited by its MVA rating. As explained earlier and described in [12], the mechanical construction of OLTC in certain transformers will limit the reverse power MVA to less than its MVA rating. DNOs should include this particular constraint based on the OLTC mechanism in the transformer. Hence forward and reverse power flow constraints are as follows,

$$
\begin{gathered}
S_{t r, f, t} \leq S_{M V A} \\
S_{t r, r, t} \leq S_{r e v, M V A}
\end{gathered}
$$

Where $S_{t r, f, t}$ and $S_{t r, r, t}$ are forward and reverse apparent power flow through transformer, $S_{M V A}$ is MVA rating of the transformer and $S_{r e v, M V A}$ is reverse power flow permissible through transformer.

Solar generation constraints: There are equality and inequality constraints associated with PV plant operational performance. The PV owner will always prefer to inject maximum possible active power as it is linked to revenues. The solar generation reactive power support will be limited by its inverter rating. Hence, the equality and inequality constraints should be considered as,

$$
\begin{gathered}
P_{\text {solar }, t}=P_{M P P T, t} \\
\sqrt{P_{\text {solar }, t}^{2}+Q_{\text {solar }, t}^{2}} \leq S_{\text {solar }} .
\end{gathered}
$$

In (7) $P_{\text {solar, },}$ and $Q_{\text {solar, },}$ are the solar active and reactive power. The solar inverter MVA rating is denoted by $S_{\text {solar }}$ and $P_{M P P T, t}$ is active power output set by MPPT. In this study, the PV is considered in the voltage control mode. It is also assumed that DNOs will be able to issue a daily voltage set point schedule for the PV plant. PV inverter overrating to offer this voltage support is considered such that PV is able to operate at 0.95 lead or lag power factor at rated active power output [9].

Tap limits: Tap changer winding has limited number of tap positions for both OLTC and VR. Typically a tap winding has a total of 32 number of steps. Each step is designed to change $0.625 \%$ of voltage. Tap changer turns ratio of each transformer must satisfy the following constraints,

$$
\text { Tap }_{\text {low }} \leq \text { Tap }_{t} \leq \text { Taphigh }
$$

In order to solve the above optimization problem described by Eq.(2)-(9), the primal-dual interior point technique is chosen due to its features such as fast convergence, efficient handling of sparsity and ease of dealing with the inequality constraints. The OLTC and PV control set points are calculated based on the day ahead irradiance and load profile. The implementation of the proposed strategy assumes that one day ahead forecast of PV and load are known with sufficient accuracy. The VR operates in autonomous mode whereas PV and OLTC in the substation have access to a communication link that can help coordinate the voltage control in the system. In the primal-dual interior point method, the inequality constraints are handled by the introduction of barrier parameter and logarithmic barrier function. In order to reduce the operation of the tap close to

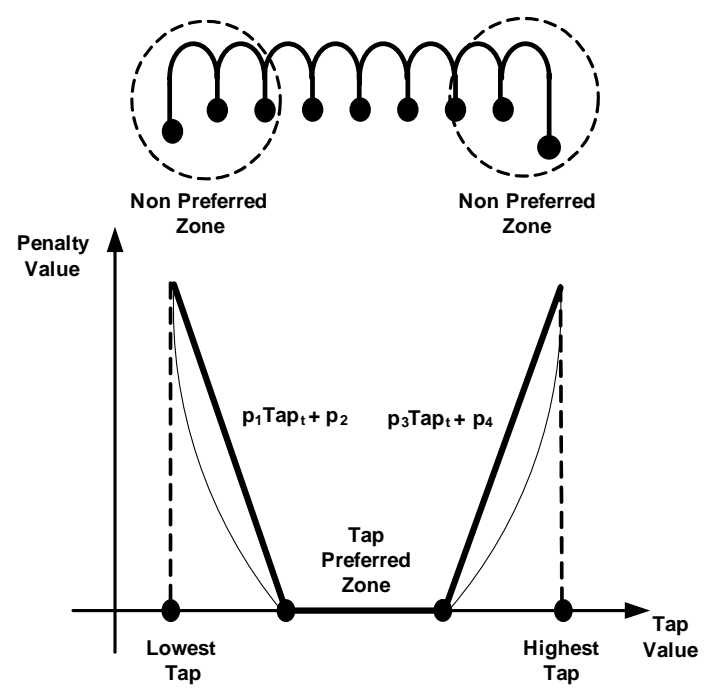

Figure 8. Proposed penalty function to avoid runaway

its limit, an additional non-preferred zone of operation around the tap limit is defined. This penalty function can be quadratic or piece wise linear as shown in Fig. 8. The piece wise linear penalty function is included in the objective function in (2) as follows,

$$
f_{\text {tap }}=W_{c} \sum_{t=2}^{t=N} \sum_{T=1}^{T=N_{\text {tr }}}\left|\operatorname{Tap}_{t, T}-\operatorname{Tap}_{t-1, T}\right|+W_{r} \sum_{t=1}^{t=N}\left|P_{t}\right|
$$

$$
P_{t} \geq 0, P_{t} \geq p_{1} \operatorname{Tap}_{t}+p_{2}, P_{t} \geq p_{3} \operatorname{Tap}_{t}+p_{4}
$$

In (10), $W_{c}$ and $W_{r}$ are scalar weights, $P_{t}$ is the penalty, $p_{1}, p_{2}, p_{3}$ and $p_{4}$ are penalty function parameters.

The proposed objective function can be combined with the conventional objective function such as power loss minimization but this work mainly focuses on tap operation counts minimization. The overall generic optimization problem formulation in the radial distribution system of Fig. 1 having OLTC, PV and autonomous VR is conveyed through a flow chart in Fig. 9. The load and solar irradiance pattern will have a major change due to change of season. The set points of communication-less devices are readjusted seasonally. The daily variations in irradiance and load are taken care of by communicating voltage set points to OLTC and PV. The next section describes a case study to demonstrate the application and effectiveness of the proposed scheme.

\section{CASE STUDY}

\section{A. System model}

The UK Generic distribution system (UKGDS) is considered. All the 95 buses in the system are at $11 \mathrm{kV}$ voltage level. The network parameters and load data are obtained from [27]. The topology of the UKGDS is shown in Fig. 10. This test system considers a typical bus which serves a mixture of four types of consumers: domestic unrestricted, domestic economy, industrial and commercial. The load profiles for each consumer class are defined in this system. The load profiles at 


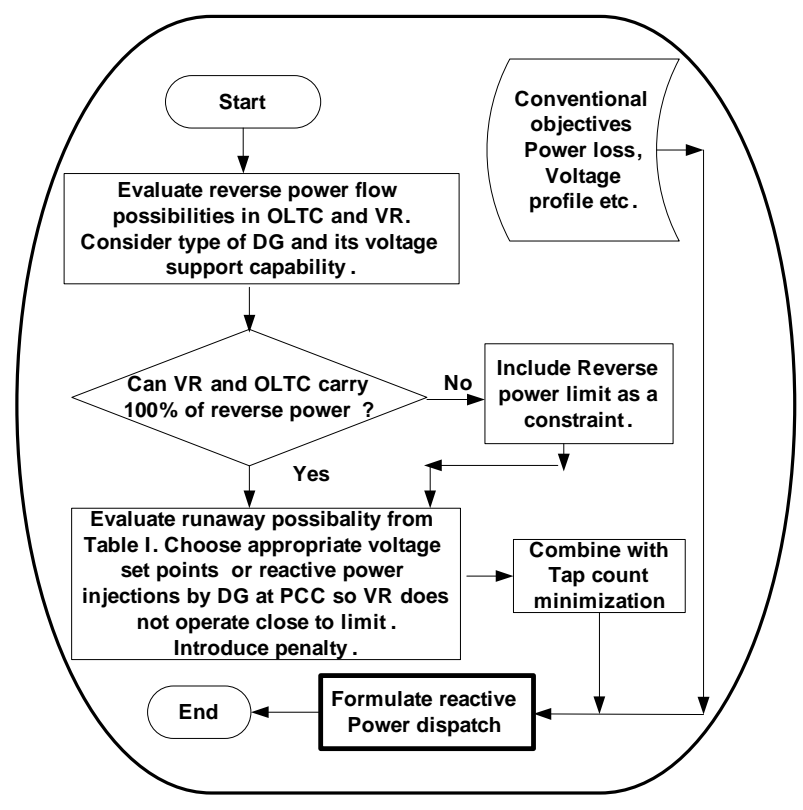

Figure 9. Problem formulation considering impact on OLTC and VR

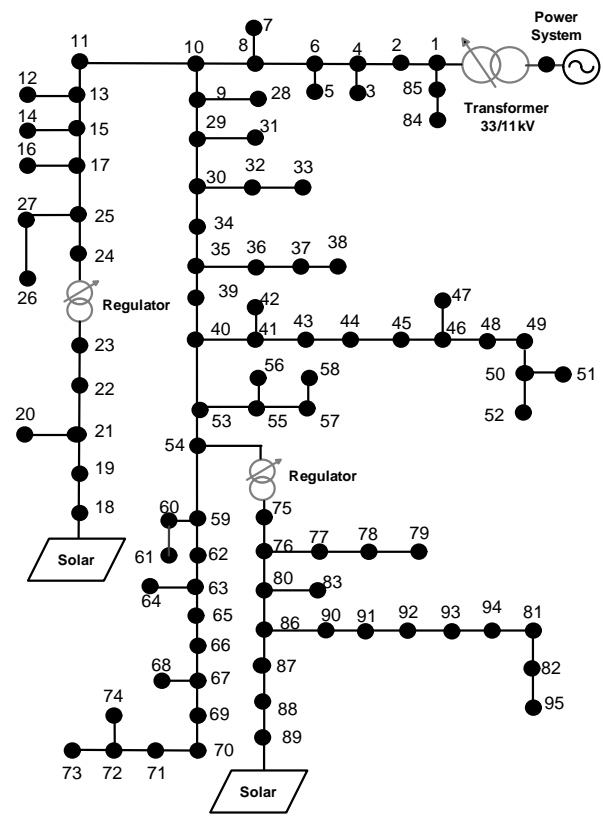

Figure 10. UKGDS 95 bus test system

different buses are generated based on the data and procedure discussed in [27]. The resultant load profile on $100 \mathrm{~kW}$ base is shown in Fig. 11. There are two PV plants considered in this system each of $1 \mathrm{MW}$ capacity. The PV plants can operate at 0.95 lead/lag power factor during peak active power injection. Appropriate inverter overrating is considered. The solar active power output profile on $100 \mathrm{~kW}$ base is shown in Fig. 12. The same irradiance profile is considered for both the plants. Half hourly load variations data are considered from [27]. The solar active power output is adopted from practical 30 second based measurements at Loughborough, England in the month of August 2012. It is assumed that at night times PV

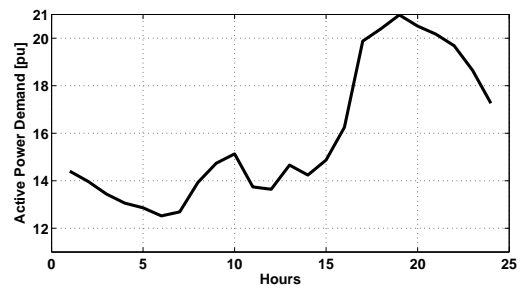

Figure 11. UKGDS system load profile

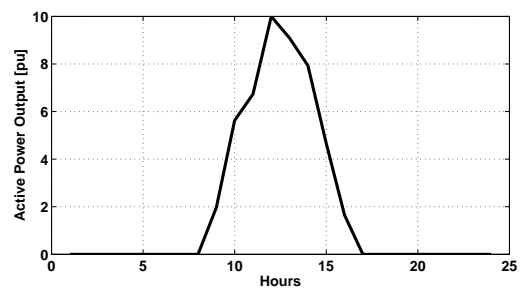

Figure 12. Solar active power profile

plant continues to offer reactive power support in a STATCOM mode. In Fig. 10, two VRs are connected between bus 24 and bus 23 and between bus 54 and 75 and control the voltage of the same buses. The system voltage values at different buses without PV are shown in Fig.13.

\section{B. Results}

The UKGDS system considers two DG at bus 18 and 89. The PV generation plants are considered at the same locations. The VR is considered in an autonomous mode and both OLTC and PV generation plants are coordinated to achieve voltage control. The objective function guarantees reduced tap counts and reduced operation of VR in non preferred zone in a preferential manner formulated using weighted combinations. The scalar weights $W_{c}$ and $W_{r}$ are varied such that $W_{c}+W_{r}=1$. Table II demonstrates simulation results for different values of weights. In Table II, VR 1 represents VR connected between bus 54 and 75, and VR 2 represents VR connected between bus 24 and 23 . Parameters for penalty function are chosen such that, the penalty function value should not be too small or too large as compared to base case tap count objective function when equal weights are assigned for both objective functions. At the end of the control range for the voltage regulator (at tap position \pm 16 ), the penalty value is significantly higher than the tap count objective function (when the weight $W_{r}$ corresponding to the voltage regulator runaway is non zero).

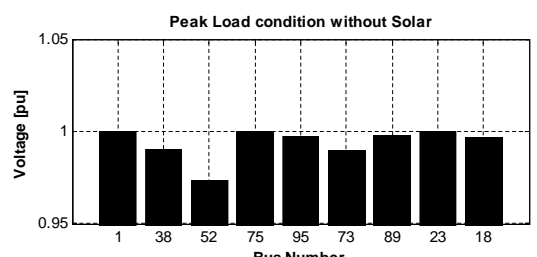

Figure 13. UKGDS system voltages during peak load condition without PV 
Table II

UKGDS 95 BUS SYSTEM

\begin{tabular}{|c|cc|cccc|cc|}
\hline & \multicolumn{2}{|c|}{ Weights } & \multicolumn{4}{c|}{ Tap Count } & \multicolumn{2}{c|}{ Operations in non preferred zone } \\
Case & Wc & Wr & OLTC & VR 1 & VR 2 & Total & VR 1 \\
\hline Case 1 & - & - & 40 & 19 & 17 & 76 & 11 & 8 \\
\hline Case 2 & 0.0 & 1.0 & 40 & 4 & 17 & 61 & 0 \\
\hline Case 3 & 1.0 & 0.0 & 29 & 2 & 2 & 33 & 9 \\
\hline Case 4 & 0.8 & 0.2 & 25 & 14 & 2 & 41 & 19 \\
\hline
\end{tabular}

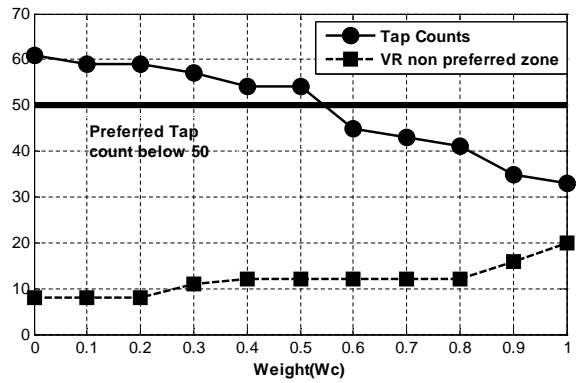

Figure 14. Variation in both objective function values at different weights

This ensures that even for the smaller value of Wr the voltage regulator operation at absolute limit is avoided. In order to maintain the control margin for the voltage regulator and avoid runaway, higher tap non-preferred zone is defined. The preferred zone for both VR is considered to be between tap positions +10 to -10 . Case 1 is considered at unity power factor non-coordinated operation. It can be observed that in Case 1 the total number of tap count is 76 and number of operations of VR1 in the non preferred zone is 11 and that for VR2 is 8 . The next case demonstrates the minimization of tap operation in the non preferred zone. The weight $W_{r}$ is set to 1 and no weight is attached to tap counts. It can be observed that all the operation of the VR1 occurs in preferred zone. But the total tap count remains 61 which is high. In the third case weight $W_{c}$ is set to 1 and no weight is attached to the penalty function. Tap counts are reduced to 33 but tap operation instances in the non preferred zone are 9 for VR1 and 12 for VR2. This case demonstrates the benefits of penalty function. Conventional objective functions do not consider the proposed penalty. It can be observed that though tap counts are reduced, tap operation happens close to limit. Case 4 gives the most satisfactory value of the objective function. The following step by step procedure is considered while tuning weights.

Step 1: There are two objectives tap counts and operation in non preferred zone. Tap count minimization is considered to be the preferred objective in this study. The weight tuning is done in preferential manner, so intuitively weight assigned for tap count minimization $W_{c}$ has to be higher.

Step 2: $W_{c}$ is set to 1 and $W_{r}$ to 0 and the best possible objective value of the tap count is calculated. Table II shows the values achieved.

Step 3: $W_{r}$ is set to 1 and $W_{c}$ to 0 and the best possible objective value of the tap in the preferred zone is calculated. Table II shows the values achieved.

Step 4: The target reduction in tap counts from the base

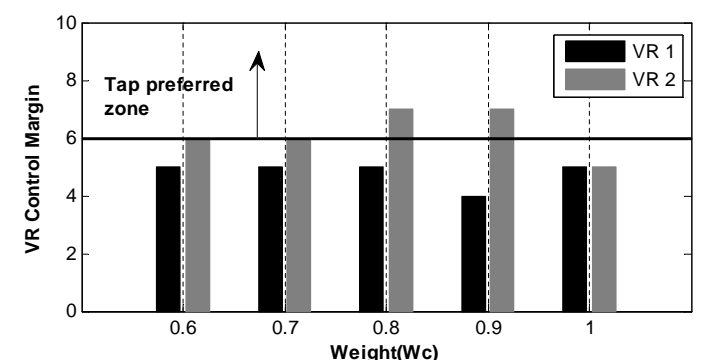

Figure 15. VR control margin at different weights

case is decided. In this simulation study it is considered 50 . Step 5: The values of $W_{c}$ are varied in steps of 0.1 from 0 to 1. The values of both objective functions are noted. Fig. 14 shows the variation in both objective function values. Case 1 tap count is 76 , considering target tap count reduction to 50 , the weight range selected is $W_{c}$ from 0.6 to 1 .

Step 6: While selecting the final value of $W_{c}$ (from 0.6 to 1 ) along with the frequency of operation of voltage regulators in non preferred zone, the voltage regulator control margin is also considered. The control margin of a voltage regulator is defined as the difference between the voltage regulator limit and the nearest operation of the voltage regulator to its limit.

Step 7: The voltage regulator preferred zone of operation is considered between tap values +10 to -10 . Hence a value above 6 is a good control margin. For every value of weight $W_{c}$ from 0.6 to 1 , the control margin for both voltage regulators is plotted. Fig. 15 demonstrates the control margin for both VR in the system.

Step 8: It can be observed from Fig. 14 and Fig. 15 that $W_{c}$ equal to 0.8 offers the same number of tap operations in non preferred zone as $W_{c}$ equal to 0.7 and the same control margin as can be achieved by $W_{c}$ equal to 0.9 , hence the final selection of weights is $W_{c}$ is equal to 0.8 and $W_{r}$ is equal to 0.2 . Thus the final optimal weight tuning is achieved for Case 4 of Table II.

Table III

Percentage Reduction In OLTC And VR TAP COUnT

\begin{tabular}{|c|c|c|c|}
\hline Case & 2 & 3 & 4 \\
\hline OLTC & $0 \%$ & $27.5 \%$ & $37.7 \%$ \\
\hline VR1+VR2 & $41.66 \%$ & $88.9 \%$ & $55.56 \%$ \\
\hline
\end{tabular}

The results of Table II are confirmed with $30 \mathrm{sec}$ time step load flow. Table III shows the percentage reduction in the OLTC and VR tap counts. Typical life of transformer and VR is 30 years. The typical maintenance interval of OLTC and VR is after 3 years. So in the base case, a total of 10 


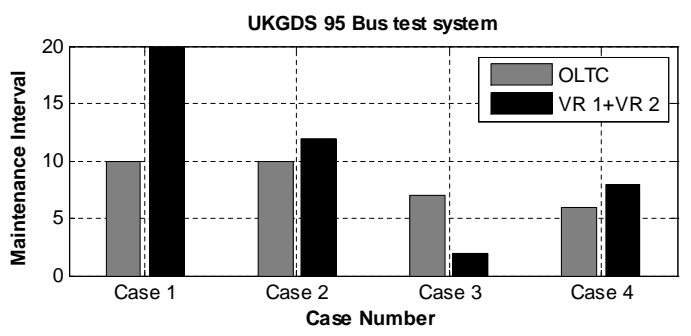

Figure 16. OLTC and VR maintenance interval
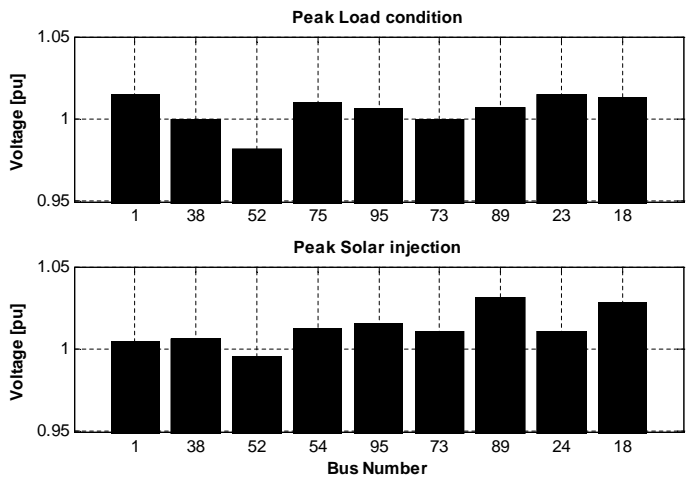

Figure 17. Voltages during peak load condition and peak solar injection

maintenance schedules are considered for each VR and OLTC. Fig. 16 shows a reduction in maintenance intervals for this test system if the daily percentage reduction as in Table III is achieved. Fig. 17 shows voltage values at different buses for Case 4 during the peak solar injection and the peak load. It can be observed that in both operating conditions, the voltage profile across the feeder is maintained within $\pm 5 \%$. Table IV shows OLTC and VR set points. The hourly voltage set points for both PV plants are shown in Fig. 18.

Table IV

OLTC AND VR SET POINTS

\begin{tabular}{|c|c|c|}
\hline & Set Points & Dead Band \\
\hline OLTC & 1.01 & 0.02 \\
\hline VR1, VR2 & 1.02 & 0.02 \\
\hline
\end{tabular}

Table V shows benefit of reactive power support from PV inverter at zero irradiance. Table $\mathrm{V}$ demonstrates Case 3 for which $W_{c}$ is 1 and $W_{r}$ is 0 . The comparison with case 3 in Table II shows that, there is significant increase in tap counts

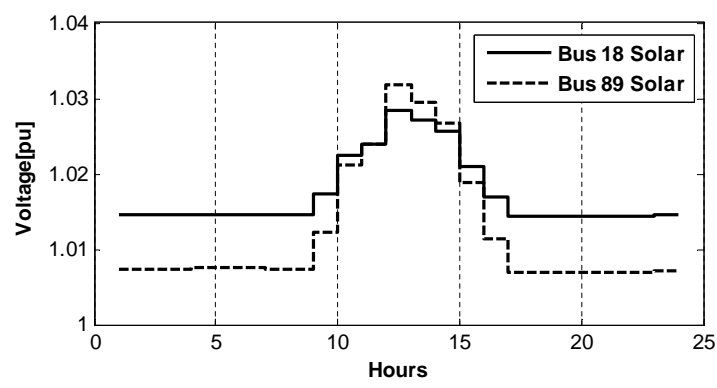

Figure 18. Hourly PV plant voltage setpoints
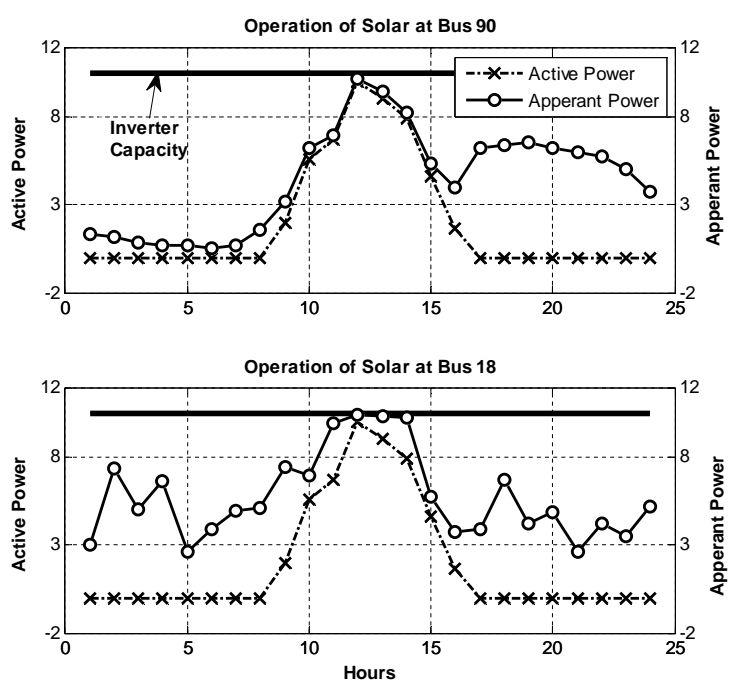

Figure 19. PV plant operation

when no reactive power support is considered from PV at zero irradiance.

Table V

TAP COUNT WITH NO REACTIVE POWER SUPPORT FROM SOLAR AT ZERO IRRADIANCE

\begin{tabular}{|c|c|c|c|}
\hline OLTC & VR1 & VR2 & Total \\
\hline 29 & 10 & 11 & 50 \\
\hline
\end{tabular}

The operation of both PV plants for a particular day under consideration is represented in Fig. 19. The active power and apparent power consumption of both the PV plants is represented in Fig. 19. The solid line represents the inverter capacity. Fig.19 demonstrates sufficient reactive power margin available at all the operating values. Also PV plant operates at MPPT at all instants. As can be observed from Fig. 18 there are total 24 optimal voltage set points calculated. The impact of PV active power variability on PCC voltage and hence on OLTC and VR is mitigated by these constant optimal voltage setpoints. On a day having higher variability frequent dispatch of PV voltage set points can be carried out by considering more number of PV active power states. This will ensure that MPPT equality constraint in (7) is enforced frequently. Even with shorter time interval, underlying principle remains the same that tap count minimization and mitigation of runaway is possible with optimum setpoint selection.

As discussed in Section I, distribution feeders are sometimes equipped with switched capacitors. The following case study demonstrates that it is possible to consider capacitors in the proposed strategy. Referring to Fig.13, it can be observed that, although the voltage at the bus 52 is within the prescribed limits, it is relatively low. Hence, at this bus, two switched capacitor banks, namely $\mathrm{C} 1$ and $\mathrm{C} 2$ each of rating $200 \mathrm{kVAr}$ at rated voltage $(1 \mathrm{pu})$, are assumed to be installed. In Europe, timer controlled switched capacitors are prevalent [20]. In order to simulate this scenario, Capacitor $\mathrm{C} 1$ is switched on at 17:00 hrs and switched off at 3:00 hrs. Capacitor C2 is switched on at 17:00 hrs and switched off at 8:00 hrs. 
Table VI

UKGDS 95 BUS SYSTEM WITH CAPACITORS AT BUS 52

\begin{tabular}{|c|cc|ccc|cc|c|}
\hline & \multicolumn{2}{|c|}{ Weights } & \multicolumn{4}{c|}{ Tap Count } & \multicolumn{3}{c|}{ Operations in non preferred zone } \\
Case & Wc & Wr & OLTC & VR 1 & VR 2 & Total & VR 1 & VR 2 \\
\hline Base Case & - & - & 31 & 21 & 17 & 69 & 5 & 8 \\
\hline Optimal Case & 0.5 & 0.5 & 27 & 16 & 2 & 45 & 6 \\
\hline
\end{tabular}

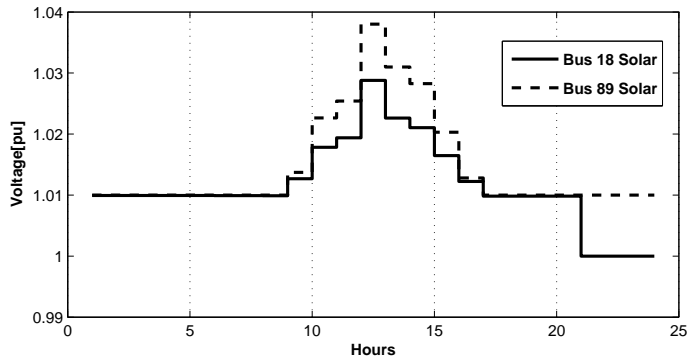

Figure 20. PV plant voltage setpoints with capacitors at bus 52
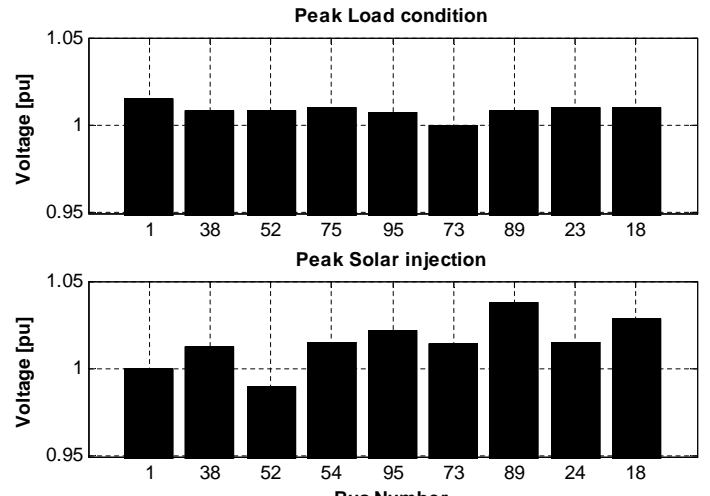

Figure 21. Voltages during peak load condition and peak solar injection with capacitors at bus 52

Table VI demonstrates simulation results for the base case and the optimal case. Fig. 20 shows the PV plant voltage set points for the optimal case. It can also be seen from Fig.21 that voltages at all buses remain within the prescribed limits.

Both case studies demonstrate that better operation of the voltage control mechanism can be achieved by using the proposed optimization procedure. Reduction in OLTC and VR maintenance cost comes at the cost of solar inverter over capacity. In cases where only one OLTC substation transformer is supplying energy to customers in a particular area, then during the OLTC maintenance it is disconnected and revenue will be lost. However, to have reactive power support from solar its inverter needs to be oversized. The cost benefit analysis needs to be carried out while planning inverter over capacity.

\section{CONCLUSIONS}

The active power injection at unity power factor by PV gives rise to over voltages. This paper has proposed a strategy that ensures smooth operation of line voltage regulators in autonomous mode. The voltage control is achieved by designing optimum voltage control set points for OLTC and PV plant.
The problem is solved by formulating weighted tap counts minimization objective subject to power flow and other constraints including limits on node voltages, tap and maximum power tracking from solar. The non preferred operating zone of VR is modeled as penalty function in the optimization process. The effectiveness of the scheme is tested through simulations on a realistic distribution network model. The case study demonstrates that with the optimal daily proposed coordinated reactive power dispatch, tap counts are reduced without detrimental impact on the feeder voltage. The operation of VR in non preferred zone is also limited and proper operation of VR without runaway is ensured. Further, definition of non preferred zone in penalty function ensures control margin for VR. It is believed that the proposed approach is useful to Distribution Network Operators to achieve voltage control in the presence of PV and autonomous VR while ensuring better operating life of OLTC and VR.

\section{ACKNOWLEDGMENT}

The authors would like to thank Dr.T.Betts from Loughborough University, UK for solar irradiance data.

\section{REFERENCES}

[1] http://www.epia.org/, "Global market outlook for photovoltaics until 2016," May 2012.

[2] C.L.Masters, "Voltage rise: the big issue when connecting embedded generation to long $11 \mathrm{kv}$ overhead lines," Power Engineering Journal, vol. 16, no. 1, pp. 5-12, 2002.

[3] R. Tonkoski, L. Lopes, and T. EL-Fouly, "Coordinated active power curtailment of grid connected pv inverters for overvoltage prevention," IEEE Trans. Sustainable Energy, vol. 2, no. 99, pp. 139-147, 2011.

[4] A. G. Madureira and J. A. P. Lopes, "Coordinated voltage support in distribution networks with distributed generation and microgrids," IET Renewable Power Generation, vol. 3, no. 4, pp. 439-454, 2009.

[5] A. G. Madureira, J. A. P. Lopes, A. Carrapatoso, and N. Silva, "The new role of substations in distribution network management," CIRED 20th Intenational Conference on Electricity Distribution, pp. 863-863, 2009.

[6] T. Stetz, F. Marten, and M. Braun, "Improved low voltage gridintegration of photovoltaic systems in germany," IEEE Trans. Sustainable Energy, vol. 4, no. 2, pp. 534-542, 2013.

[7] A. G. Madureira and J. A. P. Lopes, "Voltage and reactive power control in mv networks integrating microgrids," Proc. 2007, Int. Conf. Renewable energies and Power Quality (ICREPQ), pp. 1-5, 2007.

[8] T. Basso, J. Hambrick, and D. DeBlasio, "Update and review of ieee p2030 smart grid interoperability and ieee 1547 interconnection standards," in Innovative Smart Grid Technologies (ISGT), 2012 IEEE PES, pp. 1-7, IEEE, 2012.

[9] http://www.bdew.de, "Bdew technical guideline for generating plants connected to the medium-voltage network," May 2012.

[10] F. Katiraei and J. R. Aguero, "Solar pv integration challenges," IEEE power and energy magazine, vol. 9, no. 3, pp. 62-71, 2011.

[11] M. Coddington, B. Mather, B. Kroposki, K. Lynn, A.Razon, A.Ellis, R. Hill, T. Key, K. Nicole, and J. Smith, "Updating interconnection screens for pv system integration," Technical Paper NREL/TP 550054063, National Renewable Energy Laboratory, February 2012. Available electronically at : http://www.osti.gov/bridge. 
[12] L. M. Cipcigan and P. C. Taylor, "Investigation of the reverse power flow requirements of high penetrations of small-scale embedded generation," IET Renewable Power Generation, vol. 1, no. 3, pp. 160-166, 2007.

[13] https://solarhighpen.energy.gov/, "Us department of energy sunshot initiative," 2008

[14] R. A. Walling, R. Saint, R. C. Dugan, J. Burke, and L. A. Kojovic, "Summary of distributed resources impact on power delivery systems," IEEE Trans. on Power Delivery, vol. 23, no. 3, pp. 1636-1644, 2008.

[15] R. H. Liang and C. K. Cheng, "Dispatch of main transformer ultc and capacitors in a distribution system," IEEE Trans. Power Delivery, vol. 16, no. 4, pp. 625-630, 2001.

[16] T. Senjyu, Y. Miyazato, A. Yona, N. Urasaki, and T. Funabashi, "Optimal distribution voltage control and coordination with distributed generation," IEEE Trans. on Power Delivery, vol. 23, no. 2, pp. 1236$1242,2008$.

[17] R. W. Uluski, "VVC in the smart grid era," in IEEE Power and Energy Society General Meeting, pp. 1-7, IEEE, 2010.

[18] M. McGranaghan, T. Ortmeyer, D. Crudele, T. Key, J. Smith, and P. Barker, "Renewable systems interconnection study: Advanced grid planning and operations," Sandia National Laboratories, 2008.

[19] F. A. Viawan and D. Karlsson, "Combined local and remote voltage and reactive power control in the presence of induction machine distributed generation," IEEE Trans. Power Systems, vol. 22, no. 4, pp. 2003-2012, 2007.

[20] F. A. Viawan and D. Karlsson, "Voltage and reactive power control in systems with synchronous machine-based distributed generation," IEEE Trans. Power Delivery, vol. 23, no. 2, pp. 1079-1087, 2008.

[21] R. A. Walling and K. Clark, "Grid support functions implemented in utility-scale pv systems," in Transmission and Distribution Conference and Exposition, 2010 IEEE PES, pp. 1-5, IEEE, 2010.

[22] W. H. Kersting, Distribution system modeling and analysis. CRC press, 2002.

[23] http://www.cooperindustries.com, "Mcgraw-edison vr-32 regulators and cl-5 series control."

[24] http://www.energy.siemens.com/, "Mj-4a(tm) and mj-4b(tm) voltage regulator control panel."

[25] P. A. N. Garcia, J. L. R. Pereira, S. Carneiro Jr, V. M. daCosta, and N. Martins, "Three-phase power flow calculations using the current injection method," IEEE Trans. Power Systems, vol. 15, no. 2, pp. 508514, 2000.

[26] P. A. N. Garcia, J. L. R. Pereira, and S. Carneiro Jr, "Voltage control devices models for distribution power flow analysis," IEEE Trans. Power Systems, vol. 16, no. 4, pp. 586-594, 2001.

[27] R. Singh, B. C. Pal, and R. A. Jabr, "Distribution system state estimation through gaussian mixture model of the load as pseudo-measurement," IET Generation Transmission and Distribution, vol. 4, no. 1, pp. 50-59, 2010.

[28] E. T. Jauch, "Possible effects of smart grid functions on LTC transformers," IEEE Trans. Industry Applications, vol. 47, pp. 1013 -1021, march-april 2011.

[29] R. A. Jabr, A. H. Coonick, and B. J. Cory, "A primal-dual interior point method for optimal power flow dispatching," IEEE Trans. Power Systems, vol. 17, no. 3, pp. 654-662, 2002.

[30] S. J. Wright, Primal-dual interior-point methods, vol. 54. Society for Industrial Mathematics, 1997.

[31] W. J. Smolinski, "Equivalent circuit analysis of power system reactive power and voltage control problems," IEEE Trans. Power Apparatus and Systems, no. 2, pp. 837-842, 1981.

[32] S. Civanlar and J. J. Grainger, "Forecasting distribution feeder loads: Modeling and application to volt/var control," IEEE Trans. on Power Delivery, vol. 3, no. 1, pp. 255-264, 1988.

[33] Federal Ministry for the Environment, Nature Conservation and Nuclear Safety (BMU), Germany, "Renewable energy sources act-EEG," January 2012. Consolidated (non-binding) version of the Act available at : http://www.erneuerbare-energien.de/en.

[34] M. A. E. Kady, B. Bell, V. F. Carvalho, R. C. Burchett, H. H. Happ, and D. R. Vierath, "Assessment of real-time optimal voltage control," Power Systems, IEEE Transactions on, vol. 1, no. 2, pp. 98-105, 1986.

[35] M. Liu, S. K. Tso, and Y. Cheng, "An extended nonlinear primal dual interior-point algorithm for reactive-power optimization of large scale power systems with discrete control variables," IEEE Trans. Power Syst., vol. 17, no. 4, pp. 982-991, 2002

[36] M. B. Liu, C. A. Canizares, and W. Huang, "Reactive power and voltage control in distribution systems with limited switching operations," IEEE Trans. Power Syst., vol. 24, no. 2, pp. 889-899, 2009.
[37] S.Paudyal, C. A. Canizares, and K.Bhattacharya, "Optimal operation of distribution feeders in smart grids," IEEE Trans. Industrial Electronics, vol. 58, no. 10, pp. 4495-4503, 2011.

[38] T. E. McDermott, "Modeling pv for unbalanced, dynamic and quasistatic distribution system analysis," in Power and Energy Society General Meeting, 2011 IEEE, pp. 1-3, IEEE, 2011.

[39] M. Fila, G. A. Taylor, M. R. Irving, J. Hiscock, P. Lang, and P. Aston, "Systematic modelling and analysis of tapp voltage control schemes," in Universities Power Engineering Conference, 2007. UPEC 2007. 42nd International, pp. 349-356, IEEE, 2007.

[40] A. Malekpour and A. Pahwa, "Reactive power and voltage control in distribution systems with photovoltaic generation," in North American Power Symposium (NAPS), 2012, pp. 1-6, IEEE, 2012.

Yashodhan P Agalgaonkar (S11) received the Bachelors in Electrical Engineering degree from Walchand College of Engineering, Sangli, India, in 2003, the M.Sc. in Electrical Power Engineering from Chalmers University of Technology, Sweden, in 2006. From 2006 to 2010, he was with Crompton Greaves and with Converteam (Now, GE Energy) as a Research Engineer. Currently, he is Research Assistant and working towards his Ph.D. in the Department of Electrical and Electronic Engineering, Imperial College London, London, U.K. His research interests include power system analysis and grid integration of renewable energy sources.

Bikash C. Pal (M00, SM02, F13) received the B.E.E.(with honors) degree from Jadavpur University, Calcutta, India, the M.E. degree from the Indian Institute of Science,Bangalore, India, and the Ph.D. degree from the Imperial College London, London, U.K, in 1990, 1992, and 1999, respectively, all in electrical engineering. Currently, he is a Professor in the Department of Electrical and Electronic Engineering, Imperial College London, London, U.K. His current research interests include state estimation, power system dynamics, and Flexible ac transmission system controllers.

Rabih A. Jabr (M02, SM09) received the B.E. degree in electrical engineering (with high distinction) from the American University of Beirut, Beirut, Lebanon, in 1997 and the Ph.D. degree in electrical engineering from Imperial College London, London, U.K., in 2000. Currently, he is an Associate Professor in the Department of Electrical and Computer Engineering at the American University of Beirut. His research interests are in mathematical optimization techniques and power system analysis and computing. 